\title{
22. Approach of the relationship between fibromyalgia and bipolar disorder through an interesting case report
}

\author{
Eirini Chainaki ${ }^{1, *}$, Nektaria Polaki $^{1}$, Dimitrios Manoukakis $^{2}$
}

${ }^{1}$ Consultant Anaesthetist Venizeleio General Hospital, Heraklion, Greece; ${ }^{2}$ Medical Director Anaesthetic Department Venizeleio General Hospital, Heraklion, Greece.* irenehainaki@yahoo.gr

Introduction: $21 \%$ of patients suffering from fibromyalgia also suffer from bipolar disorder [1] and reversibly $50 \%$ of patients with bipolar disorder (BD) suffer from chronic pain syndromes including fibromyalgia [2]. Fibromyalgia and BD seem to share the same pathophysiologic mechanism [3]. The aim of this case report is to emphasize the interrelation of fibromyalgia and $\mathrm{BD}$ in order to improve the therapeutic management of those patients.

Method: A 45 years old female patient came into the Pain Clinic complaining about exacerbation of fibromyalgic pain for six months. She was receiving venflaxine $300 \mathrm{mg}$, Aripiprazole $10 \mathrm{mg}$, gabapentin $1800 \mathrm{mg}$, mirtazapine $30 \mathrm{mg}$ daily. For analgesia she was taking a combination of paracetamol/ codeine in a daily dose of $4 \mathrm{gr} / 500 \mathrm{mg}$ respectively and transdermal buprenorphine $70 \mu \mathrm{cg}$. Therapeutic interventions with trigger point injections and intra-articular injections on both knees was given. Past medical history: BD type II for 20 years, fibromyalgia for ten years, hypothyroidism. Social history: married with two children. She was working in administration of a public service. Physical examination: neck stiffness, tender points, WPI $=9$ and SSS = 7, score on PHQ-9 questionnaire 20/27. Multivariant therapeutic approach: Adjustment of fibromyalgia pharmaceutical therapy. Communication with treating psychiatrist for modification of the therapy for BD. Complementary acupuncture therapy was provided. Evaluation of patient using VAS score and dose of analgesic medication on every session and three months after therapy. Questionnaire PHQ-9 was repeated at the end of therapy and three months afterwards.

Results: There was gradual reduction on the intensity of pain. The analgesic therapy was interrupted after the third session. PHQ-9 scored 5/27 at the end of therapy and 7/27 three months later.

Conclusions: Fibromyalgia and BD can coexist and the symptoms of one may shadow or worsen the symptoms of the other. Multivariant approach is needed for effective therapy and psychiatric intervention.

\section{References}

[1] Kudlow PA, Rosenblat JD, Weissman CR, Cha DS, Kakar R, McIntyre RS, et al. Prevalence of fibromyalgia and co-morbid bipolar disorder: A systematic review and meta-analysis. The Journal of Affective Disorders. 2015; 188: 134-142.

[2] Stubbs B, Eggermont L, Mitchell AJ, De Hert M, Correll CU, Soundy A, et al. The prevalence of pain in bipolar disorder: a systematic review and large-scale meta-analysis. Acta Psychiatrica Scandinavica. 2015; 131: 75-88.

[3] Bortolato B, Berk M, Maes M, McIntyre RS, Carvalho AF. Fibromyalgia and Bipolar Disorder: Emerging Epidemiological Associations and Shared Pathophysiology. Current Molecular Medicine. 2016; 16: 119-136.

[4] Xia Y, Cao X, Wu G, Cheng J. Acupuncture Therapy For Neurological Diseases: A Neurobiological View. Springer Heidelberg Dordrecht: London New York. 2010 